\title{
Tumor Immune Cell Infiltration Patterns and Predictable Clinical Benefit with Nivolumab in Combination with Ipilimumab for 3P Implementation
}

\author{
Joong Hyuk Sheen \\ National Cancer Center Research Institute \\ Jinseon Yoo
}

Catholic University of Korea College of Medicine: Catholic University of Korea School of Medicine Tae-Min Kim

Catholic University of Korea College of Medicine: Catholic University of Korea School of Medicine Sang-Jin Lee ( $\sim$ leesj@ncc.re.kr)

National Cancer Center https://orcid.org/0000-0002-4478-1043

Ji-Youn Han

National Cancer Center Research Institute

\section{Research Article}

Keywords: CTLA-4, PD-1, immune cell biomarker, combination immunotherapy, tumor-immune microenvironment, PPPM (predictive preventive personalized medicine)

Posted Date: February 7th, 2022

DOl: https://doi.org/10.21203/rs.3.rs-986280/v1

License: (c) (i) This work is licensed under a Creative Commons Attribution 4.0 International License. Read Full License 


\section{Abstract \\ Background}

The combination immunotherapy (CIT), administering both nivolumab and ipilimumab, has demonstrated the durable efficacy in patients with skin cutaneous melanoma (SKCM), kidney renal cell carcinoma (KIRC), and lung adenocarcinoma (LUAD). However, the survival benefits of the CIT vary among responders according to tumor type and thus, the biomarker development for $\mathrm{CIT}$ is necessary for predictive, preventive, and personalized (3P) medicine.

\section{Purpose}

To understand the mechanisms underlying the differential clinical efficacy and to explore the predictive biomarkers of the CIT, we analyzed transcriptome-based immune landscapes in SKCM, KIRC, and LUAD.

\section{Methods}

We obtained bulk tumor RNA-Seq data of LUAD ( $n=517), \operatorname{KIRC}(n=506)$, and SKCM $(n=472)$ from the Cancer Genome Atlas (TCGA) consortium and examined gene signature-based tumor-infiltrating immune cell profiles, as well as the correlations among expression levels of immune checkpoints and individual tumor mutational burden (TMB).

\section{Results}

Immunoprofiling revealed three subgroups of hot, intermediate, and cold clusters according to immune cell infiltration patterns for three tumor types examined. Among the relationship between immune checkpoints, CTLA-4 and PD-1 levels from LUAD and KIRC tumors were predominantly upregulated in immune-hot subgroups and exhibited strong concordance with each other (Spearman's $r=0.75$ for LUAD; $r=0.75$ for KIRC). SKCM tumors were distinguished from LUAD and KIRC by manifesting relatively weaker correlations between PD-1 and CTLA-4 expression ( $r=0.58$ ). Further analyses in the LUAD cohort presented that expression levels of immune checkpoints were dependent upon individual patient TMB, while overall tumor-infiltrating patterns of immune cells were poorly correlated with the mutational burden except the CD56 ${ }^{\mathrm{dim}} \mathrm{NK}$ cell subset.

\section{Conclusion}

Our data suggest that the gene signature-based profiling of tumor-infiltrating immune cells guides us to a better understanding of an immune landscape of the tumor immune microenvironment (TIME), and to predict the clinically demonstrated efficacy of the CIT in each cancer type. Therefore, CIT implemented 
through a more comprehensive characterization of immune features in individual patient's tumors may enhance the clinical benefit of the predictive, preventive, personalized medicine (3PM) in cancer patients.

\section{Introduction}

Immune checkpoint inhibition, a new paradigm of targeting the PD-1/PD-L1 or CTLA-4 pathways, is regarded as the most promising cancer immunotherapeutic option [1, 2]. Although most clinical trials with immune checkpoint inhibitors have demonstrated unprecedented durable clinical responses in several types of cancers, varying efficacy of the treatment and incompletely understood resistance mechanism(s) are considered profound impediments to overcome [3]. Moreover, a wealth of data that underscores enhanced survival benefits after treatment with a combination of anti-PD- 1 and anti-CTLA-4 inhibitors compared with monotherapy [4] has nourished an increase in proposing of the combination immunotherapy (CIT) [5].

Nivolumab, a fully humanized IgG4 antibody against a PD-1, has improved outcomes of patients with several different cancer types such as melanoma, renal cell carcinoma (RCC) and non-small cell lung cancer (NSCLC) [6]. Ipilimumab, a humanized IgG1 antibody targeting CTLA-4, has been approved for the treatment of advanced melanoma [7]. A combination of CTLA-4 and PD-1/PD-L1 inhibitors is expected to increase immune cell activation in lymph nodes as well as in peripheral tissues and also to reverse $T$ cell exhaustion [8]. Thus far, nivolumab with ipilimumab is the only CIT approved for clinical use for treatment of advanced melanoma and intermediate or high-risk RCC $[9,10]$. Although this CIT has shown remarkable success in patients with advanced melanoma and RCC, variable differences in the efficacy were observed between tumors, suggesting that it needed more predictable biomarker(s) for personalized treatment. Among patients with advanced melanoma, significantly longer progression-free survival (PFS) and overall survival (OS) were observed with the CIT or with nivolumab alone than with ipilimumab alone $[5,11]$. Although the trial was not designed to compare the efficacy of CIT versus nivolumab alone, the survival outcome appeared to slightly favor the CIT over the monotherapy across clinically relevant subgroups, including the tumors with the PD-L1 expression level less than $5 \%$ or less than $1 \%[5,11]$. In addition, the $\mathrm{CIT}$ resulted in a higher objective response rate compared with nivolumab alone regardless of the tumor PD-L1 expression level. While better survival outcomes may be obtained with the CIT, the ROC-curve analyses in the study did not identify a threshold of tumor PD-L1 expression for the discrimination of a difference in overall survival $[5,11]$. Among patients with RCC, OS and response rate were significantly higher in patients treated with the CIT than with sunitinib. Meanwhile, a longer PFS was observed in PD-L1+ patients treated with the CIT compared with sunitinib but not in those with negative PD-L1 expression [12].

Tumor mutational burden (TMB) was identified as an effective biomarker for predicting patients with the highest likelihood of clinical benefit from nivolumab and ipilimumab combination in NSCLC [13, 14]. The greatest benefit of this CIT was reported in patients with high TMB, irrespective of PD-L1 expression [15], but unresolved questions regarding numerical TMB cut-off values [16] and complex TMB-“TIME” (Tumor- 
immune microenvironment) interplays as shown in patients with high TMB and insufficient inflammation [17] are still far from being completely understood.

The complexity of tumor immune microenvironment (TIME) does not permit the single factor categorization just by PD-L1/CTLA4 expression and thus, omics strategy on discovering an adequate biomarker is essential for employment of 3P medicine [18]. We herein characterized immune cell infiltrations in melanoma, RCC, and lung adenocarcinoma using bulk tumor RNA-Seq data obtained from TCGA (the Cancer Genome Atlas) database and evaluated differences in expression patterns and thus potential inhibitory mechanisms of immune checkpoints on effector $T$ cells $\left(T_{\text {eff }}\right)$ in the TIME, which could predict differential clinical efficacy of CIT and could help implementing 3P cancer immunotherapy [8].

\section{Methods}

\section{Data source}

RSEM-normalized tumor bulk RNA-Seq data for LUAD (lung adenocarcinoma, $\mathrm{N}_{\text {tumor }}=517$ ), KIRC (kidney renal cell carcinoma, $\mathrm{N}_{\text {tumor }}=506$ ) and SKCM (skin cutaneous melanoma, $\mathrm{N}_{\text {tumor }}=472$ ) cohorts (normal tissue excluded) from TCGA were downloaded from the Broad Institute Firehose pipeline (http://gdac.broadinstitute.org). Somatic mutation calls in MAF (mutation annotation format) were downloaded from TCGA portal (https://tcga-data.nci.nih.gov). Tumor purity was obtained from the PanCan ABSOLUTE purity/ploidy file (https://gdc.cancer.gov/about-data/publications/pancanatlas). For correlation analyses, we excluded one sample each from KIRC and SKCM cohorts since PD-L1 values for the samples were more than two standard deviations from the mean. Neither Spearman's $r$ values nor $p$ values were significantly affected by removal of the samples. All statistical analyses were performed with R software (https://www.r-project.org/) unless otherwise specified.

\section{Immune signature gene set}

As previously described by Şenbabaoğ lu et al. [19], our analyses of infiltration patterns of immune cells employed the 24 immune cell gene signatures from Bindea et al[20]. The signature consisted of antigen presentation machinery (APM) score, $T$ cells, central memory $T\left(T_{\mathrm{cm}}\right)$ cells, effector memory $T\left(T_{\mathrm{em}}\right), \mathrm{T}$ helper cells, type 1 helper (Th1) T cells, type 2 helper $T$ (Th2) cells, $T$ follicular helper (Tfh) cells, $T$ helper 17 (Th17) cells, regulatory $T\left(T_{\text {reg }}\right)$ cells, gd T (Tgd) cells, CD8 ${ }^{+} \mathrm{T}$ cells, cytotoxic cells, B cells, natural killer (NK) cells, CD $56^{\text {dim }}$ NK cells, CD $56^{\text {bright }}$ NK cells, dendritic cells (DC), immature DCs (iDC), plasmacytoid DCs ( $\mathrm{pDC}$ ), activated DCs (aDC), mast cells, eosinophils, macrophages, and neutrophils. In addition, our signature included normalized expression values of PD-1 (PDCD1), PD-L1 (CD274), CTLA-4 genes, as well as stromal and immune scores from the ESTIMATE algorithm [21].

\section{ssGSEA-based scoring of immune cells}


The relative levels of immune cell infiltration were quantified by a previously published computational technique that applies the expression-based immune signature gene set [20] to individual tumor samples using the single sample gene set enrichment analysis (ssGSEA) method [22]. The ssGSEA calculates separate enrichment scores for each paring of a sample and a gene set. Each ssGSEA score represents the degree to which the genes in a particular gene set are coordinately up- or down-regulated within a sample. The ssGSEA method was executed using the R package gsva (e.g. gsva(input data, signature, method="ssgsea"))[19].

\section{Unsupervised clustering and statistical tests}

Immune cell scores and gene expression levels were subject to hierarchical clustering using ward linkage and euclidean distance. Two-tailed $t$ tests were performed for continuous comparisons and Spearman's correlation test was used for the correlation analyses. To combine correlation levels of gene pairs, we employed a CLR method previously proposed by Faith et al [23]. For this, we first transformed Spearman's correlation coefficients for selected genes (i.e. PD-1 vs. CTLA-4 or PD-L1 vs. CTLA-4) into $z$ scores using Fisher transformation. Then we combined $z$ scores using a formulation of $\operatorname{sqrt}\left(\mathrm{z}_{1}{ }^{2}+\mathrm{z}_{2}{ }^{2}\right)$ as previously described by Faith et al[23]. Spearman's correlation was applied to evaluate associations among genomic features in this study except analyses involving correlation between TMB $v s$. residuals immune cell scores which employed partial Spearman's correlation corrected for tumor purity. As previously demonstrated by others [24] the residuals of the immune scores derived from the regression plots of tumor purity were correlated with TMB.

\section{Results}

\section{Tumor-immune microenvironment (TIME) analyses in LUAD}

RNA-Seq data of LUAD, SKCM, and KIRC were analyzed for the transcriptome-based immunoprofiling of the TIME across tumor types [19]. Relative tumor-infiltrating levels of immune cells were estimated in silico using previously published single sample gene set enrichment analysis (sSGSEA)-based approaches. Along with immune signature scores, expression values of three immunotherapy targets (PD1, PD-L1, CTLA-4), as well as ESTIMATE algorithm-based total immune/stromal scores [21] were also estimated for LUAD. The TIME profile was then subject to hierarchical clustering for immune-based taxonomy (Fig. 1A-B). A heatmap result showed samples were largely segregated into three subgroups according to immune infiltration patterns. A "hot" subgroup ( $n=136,26.3 \%)$, termed for highly inflamed tumors with elevated levels of immune infiltrations $[3,25]$, was enriched in CD56 dim NK cells, cytotoxic cells, CD8 ${ }^{+} T$ cells, B cells, Th1, as well as regulatory $T$ cells $\left(T_{\text {reg }}\right)$. An "intermediate" subgroup $(n=163$, 31.5\%) manifested a heterogeneous presence of immune cells at varying levels with intact infiltrations of macrophages, dendritic cells (DC), neutrophils, central and effector memory $T$ cells $\left(T_{\mathrm{cm}}, T_{\mathrm{em}}\right.$ respectively). An immunologically "cold" subgroup ( $n=218,42.2 \%)$ [26] was dominantly immuneexcluded, exhibited the lowest antigen presentation machinery (APM) score and nominal infiltration of 
immune cells except Th17, Th2 and CD56 ${ }^{\text {bright }}$ NK cells (Fig. 1A). To broadly assess potential inhibitory mechanisms in this cohort, we evaluated immune checkpoint gene expression based on the aforementioned TIME subtypes (Fig. 1C-F). PD-1 and CTLA-4 were remarkably upregulated in the hot cluster, closely heeding distribution of tumor-infiltrating $C D 8^{+} T$ cells, $T_{\text {reg, }}$ cytotoxic cells and CD $56^{\mathrm{dim}} \mathrm{NK}$ cells, whereas their levels stagnated in the intermediate and cold subgroups. The PD-L1 level was highest in the hot subgroup and showed a steady decline in the intermediate and cold subgroups. To interrogate the relationship between expression levels of immune checkpoints in the TIME context, we conducted correlation analyses with PD-1, CTLA-4, and PD-L1 in the LUAD cohort. The expression levels of CTLA-4 and PD-1 were strongly related (Spearman's $r=0.75$, p<2.2e-16) (Fig. 1G) while correlation of CTLA-4 and PD-L1 was moderate (Spearman's $r=0.52, p<2.2 e-16$ ) (Supplementary Fig. 1A). The normalized correlation level[23] between CTLA-4 and PD-1 was also stronger ( $z$ score $=6.194)$ than that of CTLA-4 and PD-L1 ( $z$ $=4.014)$. Taken together, our results may suggest the CIT could be more beneficial in LUAD patients belonging to the hot subgroup with copious immune cell infiltrations and strong concurrent expression among the immune checkpoints.

\section{Tumor-immune Microenvironment (Time) Analyses In Kirc}

When we assessed a KIRC cohort employing the same computational methodologies, patients were similarly segregated into three TIME subgroups (Fig. 2A-B). Various $T_{\text {eff }}$ and $B$ cells were enriched predominantly in the hot subgroup $(n=114,22.5 \%)$, and as infiltrations of the majority of $T$ cells decreased, certain immune cell types (Th17, neutrophils, and DCs) remained prevalent in the intermediate group ( $n=267,52.8 \%$ ). The cold subgroup $(n=125,24.7 \%)$ showed similar ESTIMATE immune scores to the intermediate ( $p>0.05$, Fig. 2B), but the cold subgroup presented poorer infiltrations of $\mathrm{CD}^{+} \mathrm{T}$ cells, memory $T$ cells and higher infiltration of $T_{\text {reg }}$, NK cells or $B$ cells compared with the intermediate subgroup. In the KIRC cohort, PD-1 and CTLA-4 were highly upregulated in the hot subgroup and downregulated the most in the intermediate group (Fig. 2C-F). In contrast, PD-L1 expression was dispersed throughout the cohort and showed modest upregulation in the hot subgroup (Fig. 2E). When the relationship between PD-1 vs. CTLA-4 (Fig. 2G) was analyzed, expression of these two genes displayed a strong correlation (Spearman's $r=0.75, p<2.2 e-16$; normalized $z$ score $=5.168$ ), whereas PD-L1 and CTLA-4 did not (Spearman's $r=0.25, p=1.5 e-08 ; z$ score=1.710) (Supplementary Fig. 1B). Similar to LUAD, our results imply patients belonging to the immune-hot subgroups with concordant overexpression of PD-1 and CTLA-4 may more likely benefit from the CIT than those of the intermediate or cold subgroup.

\section{Tumor-immune Microenvironment (Time) Analyses In Skcm}

Three immune-subgroups were also observed for the SKCM cohort (Fig. 3A-B) after unsupervised clustering of the TIME profiles. The first subgroup signifying a hot TIME $(n=88,18.6 \%)$ showed the highest infiltration of $\mathrm{CD} 8^{+} \mathrm{T}$ cells, Th1, CD56 dim NK cells, cytotoxic cells, $\mathrm{T}_{\text {reg, }}$, as well as the majority of antigen presenting cells (APC) into tumors. The intermediate group $(n=221,46.8 \%)$ showed lower degrees 
of infiltration compared with the hot group, while the cold group was almost completely immuneexcluded with few exceptions (Th2, eosinophils and $T_{\mathrm{cm}}$ among others) $(\mathrm{n}=163,34.5 \%)$. We documented PD-1, CTLA-4 and PD-L1 were largely upregulated in the hot subgroup but downregulated the most in an immune-excluded cold subgroup (Fig. 3C-F). While CTLA-4 levels between the intermediate and the cold subgroups were different with statistical significance $(p=0.036)$, the difference was much less dramatic compared with PD-1 ( $<<2.2 \mathrm{e}-16)$, suggesting the possibility that CTLA-4 is not expressed in strong accordance with PD-1 or with the TIME context (Fig. 3C-D). This discordance unseen in previous tumor types was further corroborated when correlation analyses were conducted with the expression levels of immune checkpoints. The correlation between PD-1 vs. CTLA-4 (Spearman's $r=0.58, p<2.2 e-16$, normalized $z$ score $=4.306$; Fig. $3 G$ ) in the SKCM cohort was relatively weaker than the correlations documented in LUAD ( $z$ score=6.194) and KIRC ( $z$ score=5.168) (Fig. 1G and Fig. $2 \mathrm{G}$ respectively). The discordance between CTLA-4 and PD-1 levels in SKCM may explain previously reported survival outcome which slightly favored CIT over monotherapy across clinically relevant subgroups [11].

\section{Tumor mutational burden (TMB) as an independent measure depicting TIME of LUAD}

Recent studies have illustrated that the expression levels of immune-related genes [27, 28], degree of $\mathrm{CD}^{+}$tumor-infiltrating lymphocytes and TMB could be employed for predicting the clinical efficacy of immunotherapy in NSCLC $[12,29]$. Building upon these findings, we evaluated correlations among the genomic markers of the LUAD cohort in the context of the TIME immune-subgroups. Nonsynonymous TMB values (Fig. 4A-B), ssGSEA-based T cell scores (Fig. 4C-D), or ESTIMATE immune scores (Supplementary Fig. 2B\&Fig. 1B) were ordered according to the unsupervised clustering result shown in Fig. 1A. While T cell or immune scores were arranged as anticipated (e.g. highest in the hot subgroup and lowest in the cold subgroup), TMB sank in the intermediate subgroup and the level was comparable between the hot and cold subgroups ( $p>0.05$; Fig. 4B), yielding the weak correlations with immuneinfiltration patterns (Supplementary Fig. 2C-D). To more broadly investigate correlations between TMB and the estimated levels of 24 immune cells, we selected marker genes specific for immune cell types, and performed correlative analyses for each gene with TMB. While associations between TMB and expression of most of the marker genes for the immune cells were negligible, the genes specific for CD56 ${ }^{\text {dim }}$ NK cells, T helper cells, cytotoxic cells or activated DC (aDC) were positively correlated with TMB (Fig. 4E).

Previous reports have unraveled confounding impacts of tumor purity on TMB evaluation and the expression pattern of immune-related genes[30]. We documented that the purity of the LUAD tumors was inversely correlated with immune scores, stromal scores or infiltration of cytolytic NK cells, and that the tumor purity was not significantly correlated with TMB (Supplementary Fig. 3). To account for the tumor purity as confounding factors, it was demonstrated through partial Spearman's correlation analyses that the levels of most tumor-infiltrating immune cells were negatively associated with TMB except APM (Spearman's $r=0.125, p=0.006)$, Th2 $(r=0.361, p=3.4 e-16)$ and CD56 $\operatorname{dim}$ NK cells $(r=0.216, p=1.77 e-06)$ (Fig. 4F). Taken together, our data reflect the highly variable and independent dynamics between TMB and immune cell-mediated inflammatory responses across LUAD patients (Fig. 4G). 


\section{CTLA-4 and PD-1 are strongly associated in LUAD regardless of TMB}

To further elucidate whether expression of different immune checkpoints are dependent upon TMB in LUAD, we divided the LUAD cohort into two groups: $T_{M B}{ }^{\text {High }}$ (TMB>median $(=207.5)$ ) and TMB ${ }^{\text {Low }}$ ( $T M B \leq$ median) (Supplementary Fig. 2A). Immune checkpoint expression was upregulated in the TMB High group ( $\mathrm{p}<0.05$; Fig. 5A-C) and TMB was weakly associated with expression of PD-1 and PD-L1 (Fig. 5D-F). The result was consistent when the expression levels of the immune checkpoints were adjusted for tumor purity: TMB was correlated with PD-L1 $(r=0.172, p=0.0002)$ and PD-1 $(r=0.148, p=0.001)$ but marginally with CTLA-4 ( $r=0.092, p=0.042)$ (Fig. 4F). We then investigated whether correlation between each immune checkpoint depends on TMB. Our results exhibited strong associations between CTLA-4 vs. PD-1 (Spearman's $r=0.74$ for TMB ${ }^{\text {High }} ; \mathrm{r}=0.75$ for TMB ${ }^{\text {Low; }}$, both $p<2.2 \mathrm{e}-16$ ) and moderate relationships between CTLA-4 vs. PD-L1 (Spearman's $r=0.53$ for TMB ${ }^{\text {High; }} r=0.5$ for TMB ${ }^{\text {Low; }}$, both $\mathrm{p}<2.2 \mathrm{e}-16$ ) and PD-1 vs. PD-L1 (Spearman's $r=0.56$ for TMB ${ }^{\text {High, }} ;=0.49$ for TMB ${ }^{\text {Low; }}$, both $p \leq 1.1 \mathrm{e}-15$ ), regardless of TMB (Fig. 5G-I). Altogether, our findings highlight the notion that the TIME context is independent of TMB [17] and that measuring TMB alone cannot serve as an established biomarker to predict $\mathrm{T}$ cell activation status in the TIME and thus sensitivity of the CIT in LUAD.

\section{Discussion}

Both CTLA-4 and PD-1 play an inhibitory role in T cell activity to maintain immune tolerance, but they are different in several aspects [31]. CTLA-4, found exclusively on T cells, signals during a priming phase of T cells in lymph nodes while PD-1, expressed on T cells, NK cells, B cells and myeloid cells, functions during an effector phase in peripheral tissues. The B7 ligand for CTLA-4 is confined to APC, whereas the ligand for PD-1, PD-L1, is expressed on leukocytes and tumors in peripheral tissues [31]. The differences in the nature of immune inhibition between them suggest that CIT would be synergistic upon clinical implementation and that tumors with elevated levels for both CTLA-4 and PD- 1 would be more efficaciously deterred by immune responses unleashed by CIT. Our computational analyses revealed hot subgroups of three tumor types and a fraction of intermediate subgroups that are consistently enriched with $\mathrm{T}_{\text {eff }}$ and NK cells, as well as the highest levels of immune checkpoints with a solid concordance of expression patterns. As might be expected, tumors as such manifest inflammatory but a highly exhausted TIME, making them potentially the most ideal group for the CIT [32, 33]. In contrast, the cold subgroups in the three cancer cohorts examined in this study displayed lowest immune cell infiltrations indicative of potentially insufficient benefits from the CIT. The tumors like this would rather require preceding immune-invigorating stimuli eliciting exposure of tumor neoantigens followed by recruitment of APC and T cells for further anti-tumor responses. Overall, immunotherapy such as CIT needs to be more aggressive 3P implementation for benefitting more cancer patients by analyzing immune cell infiltration in TIME and significantly cutting the enormous costs of treating cancer patients.

CTLA-4 is induced upon activation of naïve $T$ cells or constitutively expressed on $T_{\text {reg, }}$, mediating an immunoregulatory role in most cancers through $\mathrm{T}_{\text {eff }}$ suppression [34]. As long as a tumor bears a 
predominance of CTLA-4 ${ }^{\text {High }}$ T cells, CIT that suppresses $\mathrm{T}_{\text {reg }}$ and re-activates exhausted $\mathrm{T}_{\text {eff }}$ would be synergistic for anti-cancer immunity, whereas tumors with fewer infiltrates or/and non-concurrent expression between $\mathrm{T}_{\text {reg }}$ vs. CTLA-4 may respond to CIT similar to nivolumab alone. Thus, we focused on how closely PD-1 or CTLA-4 is related to immune infiltrates (e.g. $T_{\text {reg }}$ ) in the LUAD, KIRC and SKCM cohorts. PD-1 expression was strongly correlated with cytotoxic cells, as well as with $\mathrm{T}_{\text {reg }}$ in all cohorts ( $T_{\text {reg }}$ vs. PD-1: Spearman's $r_{\text {LUAD }}=0.674 ; r_{K I R C}=0.646 ; r_{S K C M}=0.721$, Supplementary Table 1 ) as reported previously $[35,36]$. CTLA-4 was highly correlated with $\mathrm{T}_{\text {reg }}$ distribution in LUAD and KIRC (Spearman's $r=0.720$ and $r=0.689$, respectively), whereas it showed relatively weaker correlation with $T_{\text {reg }}$ in SKCM (Spearman's $r=0.459$ ) (Supplementary Table 2). Instead, CTLA-4 was more strongly correlated with Th1 cells in SKCM ( $r=0.604)$, implying a possibility that higher CTLA-4 expression more likely represent exhausted $\mathrm{T}_{\text {eff }}$ in SKCM. Along with an observation that anti-CTLA-4 blockade induces specific expansion of ICOS ${ }^{+} \mathrm{TBET}^{+}$Th1-like $\mathrm{CD} 4^{+} \mathrm{T}$ cells in melanoma patients [37] and a finding that PD-L1 upregulation in melanoma cells is regulated by interferon gamma exposure which could predominantly be elicited by Th1 cells [38, 39], our result prompted us to speculate that CIT would still be beneficial compared with monotherapy for all SKCM subgroups.

Although it was recently demonstrated TMB did not correlate strongly with response to CIT in melanoma patients [40], TMB has been highlighted as a predictive marker of efficacy in a dataset of NSCLC and SCLC patients treated with CIT $[15,41]$. In the LUAD cohort, TMB was comparably high in the immuneexcluded cold subgroup as the inflamed, hot subgroup, and overall immune infiltrations were not substantially correlated with TMB (Fig. 4), implicating that other predictable biomarker would be needed like the overall signaling pathway network profiling [42] and plasma complement C7 [43]. We also reported that few immune cell-specific (e.g. cytotoxic cells, CD56 dim NK cells, T helper cells) marker genes were correlated with TMB (Fig. 4E). However, when the residuals of the immune infiltrate scores derived from the regressions plots of tumor purity were correlated with TMB, CD56 ${ }^{\mathrm{dim}}$ NK cells were the only immune feature with the consistent positive correlation with TMB (Fig. 4F). The observation here not only illuminates the need to account for tumor purity during in silico immunoprofiling but also calls for further functional and clinical characterization of the NK cell subsets in the TIME, facilitating their potential role as a predictable biomarker for CIT in the combination with TMB in LUAD.

While we depicted that the levels of immune checkpoints are dependent upon TMB in the LUAD, greater correlations were revealed in the same cohort between PD-1 vs. CTLA-4 regardless of TMB which does not closely follow infiltration patterns of anti-tumor immune cells. Thus, our findings unveiled the highly heterogeneous nature of the TIME where discrepancies between loads of somatic mutation which may produce neoantigens and the levels of $T$ cell-mediated anti-tumor responses elicited by the antigens exist (Fig. 4G). As long as tumor-intrinsic antigen presentation and cytolytic activity exerted by immune infiltrates are indispensable in determining the outcome of immunotherapy, our result underscores that treatment sensitivity is not solely dependent upon individual patient's TMB nor expression levels of a single immune checkpoint molecule (e.g. PD-L1) [44]. Supporting this, TMB and T cell-inflamed gene expression profile have independently predicted treatment response with low correlation to each other in 
HNSCC and melanoma patients [17], and genomic expression related to immune checkpoints [1, 45, 46] or cytolytic activity $[47,48]$ has been diversely proposed to be potential predictive markers for immunotherapy.

\section{Conclusions}

Our approaches employing estimation of tumor-infiltrating immune cell patterns to dissect landscapes of the patient individual TIME could potentially guide us for a better understating of the crosstalk among immune checkpoints, immune infiltrates and the TMB, which could predict personalized clinical benefits with the CIT. Together with more refined TMB and PD-1/PD-L1 biomarker criteria, the data presented here bolster the need to implement 3P strategies by incorporating transcriptome-oriented correlative analyses of druggable immune-targets in the context of immune cell infiltration signatures in the TIME.

\section{List Of Abbreviations}

3PM (predictive preventive personalized medicine), Combination immunotherapy (CIT), the Cancer Genome Atlas (TCGA), skin cutaneous melanoma (SKCM), kidney renal cell carcinoma (KIRC), lung adenocarcinoma (LUAD), renal cell carcinoma (RCC), non-small cell lung cancer (NSCLC), tumor-immune microenvironment (TIME), tumor mutational burden (TMB), progression-free survival (PFS), overall survival (OS), effector $T$ cells $\left(T_{\text {eff }}\right)$, regulatory $T$ cells $\left(T_{\text {reg }}\right)$, single sample gene set enrichment analysis (ssGSEA), Estimation of STromal and Immune cells in MAlignant Tumor tissues using Expression data (ESTIMATE).

\section{Declarations}

\section{Authors' contributions}

J-Y.H. and T-M.K. conceptually designed the study. J.H.S. and J .Y. collected and generated the data analyzed for this study. All authors contributed to data analysis and interpretation. J.H.S., S-J.L., and JY.H. were major contributors in writing the manuscript. All authors read and approved the final manuscript and are accountable for all aspects of the work.

\section{Funding}

This study was supported by a grant from the National Cancer Center, Korea (NCC-1610281) and a grant from the Ministry of Science and ICT through the NRF, Korea (No. 1731530).

\section{Compliance with ethical standards}

Competing interests: The authors declare that they have no competing interests.

Ethics approval and consent to participate: Not applicable. 
Consent for publication: Not applicable.

Availability of data and material: All data generated or analyzed during this study are included in this published article and in its supplementary data.

\section{References}

1. Taube JM, et al. Association of PD-1, PD-1 ligands, and other features of the tumor immune microenvironment with response to anti-PD-1 therapy. Clin Cancer Res. 2014;20(19):5064-74.

2. Liskova A, et al., Flavonoids as an effective sensitizer for anti-cancer therapy: insights into multifaceted mechanisms and applicability towards individualized patient profiles. EPMA J, 2021: p. 122.

3. Tray N, Weber JS, Adams S. Predictive Biomarkers for Checkpoint Immunotherapy: Current Status and Challenges for Clinical Application. Cancer Immunol Res. 2018;6(10):1122-8.

4. Baumeister $\mathrm{SH}$, et al. Coinhibitory Pathways in Immunotherapy for Cancer. Annu Rev Immunol. 2016;34:539-73.

5. Larkin J, et al. Combined Nivolumab and Ipilimumab or Monotherapy in Untreated Melanoma. N Engl J Med. 2015;373(1):23-34.

6. Motzer RJ, et al. Nivolumab for Metastatic Renal Cell Carcinoma: Results of a Randomized Phase II Trial. J Clin Oncol. 2015;33(13):1430-7.

7. Wolchok JD, et al. Nivolumab plus ipilimumab in advanced melanoma. $\mathrm{N}$ Engl $\mathrm{J}$ Med. 2013;369(2):122-33.

8. Rotte A, Jin JY, Lemaire V. Mechanistic overview of immune checkpoints to support the rational design of their combinations in cancer immunotherapy. Ann Oncol. 2018;29(1):71-83.

9. Hodi FS, et al., Nivolumab plus ipilimumab or nivolumab alone versus ipilimumab alone in advanced melanoma (CheckMate 067): 4-year outcomes of a multicentre, randomised, phase 3 trial. Lancet Oncol, 2018.

10. Motzer RJ, et al. Nivolumab plus Ipilimumab versus Sunitinib in Advanced Renal-Cell Carcinoma. N Engl J Med. 2018;378(14):1277-90.

11. Wolchok JD, et al. Overall Survival with Combined Nivolumab and Ipilimumab in Advanced Melanoma. N Engl J Med. 2017;377(14):1345-56.

12. Miura Y, Sunaga N. Role of Immunotherapy for Oncogene-Driven Non-Small Cell Lung Cancer. Cancers (Basel), 2018. 10(8).

13. Hellmann MD, et al. Nivolumab plus ipilimumab as first-line treatment for advanced non-small-cell lung cancer (CheckMate 012): results of an open-label, phase 1, multicohort study. Lancet Oncol. 2017;18(1):31-41.

14. Hellmann MD, et al. Nivolumab plus Ipilimumab in Lung Cancer with a High Tumor Mutational Burden. N Engl J Med. 2018;378(22):2093-104. 
15. Hellmann MD, et al. Genomic Features of Response to Combination Immunotherapy in Patients with Advanced Non-Small-Cell Lung Cancer. Cancer Cell. 2018;33(5):843-52 e4.

16. Rizvi NA, et al. Cancer immunology. Mutational landscape determines sensitivity to PD-1 blockade in non-small cell lung cancer. Science. 2015;348(6230):124-8.

17. Cristescu R, et al., Pan-tumor genomic biomarkers for PD-1 checkpoint blockade-based immunotherapy. Science, 2018. 362(6411).

18. Aydin B, Caliskan A, Arga KY. Overview of omics biomarkers in pituitary neuroendocrine tumors to design future diagnosis and treatment strategies. EPMA J. 2021;12(3):383-401.

19. Senbabaoglu Y, et al. Tumor immune microenvironment characterization in clear cell renal cell carcinoma identifies prognostic and immunotherapeutically relevant messenger RNA signatures. Genome Biol. 2016;17(1):231.

20. Bindea G, et al. Spatiotemporal dynamics of intratumoral immune cells reveal the immune landscape in human cancer. Immunity. 2013;39(4):782-95.

21. Yoshihara K, et al. Inferring tumour purity and stromal and immune cell admixture from expression data. Nat Commun. 2013;4:2612.

22. Barbie DA, et al. Systematic RNA interference reveals that oncogenic KRAS-driven cancers require TBK1. Nature. 2009;462(7269):108-12.

23. Faith JJ, et al. Large-scale mapping and validation of Escherichia coli transcriptional regulation from a compendium of expression profiles. PLoS Biol. 2007;5(1):e8.

24. Li B, et al. Comprehensive analyses of tumor immunity: implications for cancer immunotherapy. Genome Biol. 2016;17(1):174.

25. Gajewski TF. The Next Hurdle in Cancer Immunotherapy: Overcoming the Non-T-Cell-Inflamed Tumor Microenvironment. Semin Oncol. 2015;42(4):663-71.

26. Haanen J. Converting Cold into Hot Tumors by Combining Immunotherapies. Cell. 2017;170(6):1055-6.

27. Rhee JK, et al. Impact of Tumor Purity on Immune Gene Expression and Clustering Analyses across Multiple Cancer Types. Cancer Immunol Res. 2018;6(1):87-97.

28. Aran D, Sirota M, Butte AJ. Systematic pan-cancer analysis of tumour purity. Nat Commun. 2015;6:8971.

29. Schalper KA, et al., Objective measurement and clinical significance of TILs in non-small cell lung cancer. J Natl Cancer Inst, 2015. 107(3).

30. Carter SL, et al. Absolute quantification of somatic DNA alterations in human cancer. Nat Biotechnol. 2012;30(5):413-21.

31. Buchbinder El, Desai A. CTLA-4 and PD-1 Pathways: Similarities, Differences, and Implications of Their Inhibition. Am J Clin Oncol. 2016;39(1):98-106.

32. Spranger $S$, et al. Mechanism of tumor rejection with doublets of CTLA-4, PD-1/PD-L1, or IDO blockade involves restored IL-2 production and proliferation of CD8(+) T cells directly within the 
tumor microenvironment. J Immunother Cancer. 2014;2:3.

33. Tumeh PC, et al. PD-1 blockade induces responses by inhibiting adaptive immune resistance. Nature. 2014;515(7528):568-71.

34. Qureshi OS, et al. Trans-endocytosis of CD80 and CD86: a molecular basis for the cell-extrinsic function of CTLA-4. Science. 2011;332(6029):600-3.

35. Lowther DE, et al., PD-1 marks dysfunctional regulatory T cells in malignant gliomas. JCI Insight, 2016. 1(5).

36. Toker A, et al. Regulatory T Cells in Ovarian Cancer Are Characterized by a Highly Activated Phenotype Distinct from that in Melanoma. Clin Cancer Res. 2018;24(22):5685-96.

37. Wei SC, et al. Distinct Cellular Mechanisms Underlie Anti-CTLA-4 and Anti-PD-1 Checkpoint Blockade. Cell. 2017;170(6):1120-33 e17.

38. Garcia-Diaz A, et al. Interferon Receptor Signaling Pathways Regulating PD-L1 and PD-L2 Expression. Cell Rep. 2017;19(6):1189-201.

39. Schoenborn JR, Wilson CB. Regulation of interferon-gamma during innate and adaptive immune responses. Adv Immunol. 2007;96:41-101.

40. Goodman AM, et al. Tumor Mutational Burden as an Independent Predictor of Response to Immunotherapy in Diverse Cancers. Mol Cancer Ther. 2017;16(11):2598-608.

41. Hellmann MD, et al. Tumor Mutational Burden and Efficacy of Nivolumab Monotherapy and in Combination with Ipilimumab in Small-Cell Lung Cancer. Cancer Cell. 2018;33(5):853-61 e4.

42. Li N, Zhan X. Signaling pathway network alterations in human ovarian cancers identified with quantitative mitochondrial proteomics. EPMA J. 2019;10(2):153-72.

43. Park JG, et al. Plasma complement $C 7$ as a target in non-small cell lung cancer patients to implement 3P medicine strategies. EPMA J. 2021;12(4):629-45.

44. Li J, et al. Tumor Cell-Intrinsic Factors Underlie Heterogeneity of Immune Cell Infiltration and Response to Immunotherapy. Immunity. 2018;49(1):178-93 e7.

45. Danilova L, et al. Association of PD-1/PD-L axis expression with cytolytic activity, mutational load, and prognosis in melanoma and other solid tumors. Proc Natl Acad Sci U S A. 2016;113(48):E776977.

46. Van Allen EM, et al. Genomic correlates of response to CTLA-4 blockade in metastatic melanoma. Science. 2015;350(6257):207-11.

47. Rooney MS, et al. Molecular and genetic properties of tumors associated with local immune cytolytic activity. Cell. 2015;160(1-2):48-61.

48. Ock CY, et al. Pan-Cancer Immunogenomic Perspective on the Tumor Microenvironment Based on PD-L1 and CD8 T-Cell Infiltration. Clin Cancer Res. 2016;22(9):2261-70.

\section{Figures}




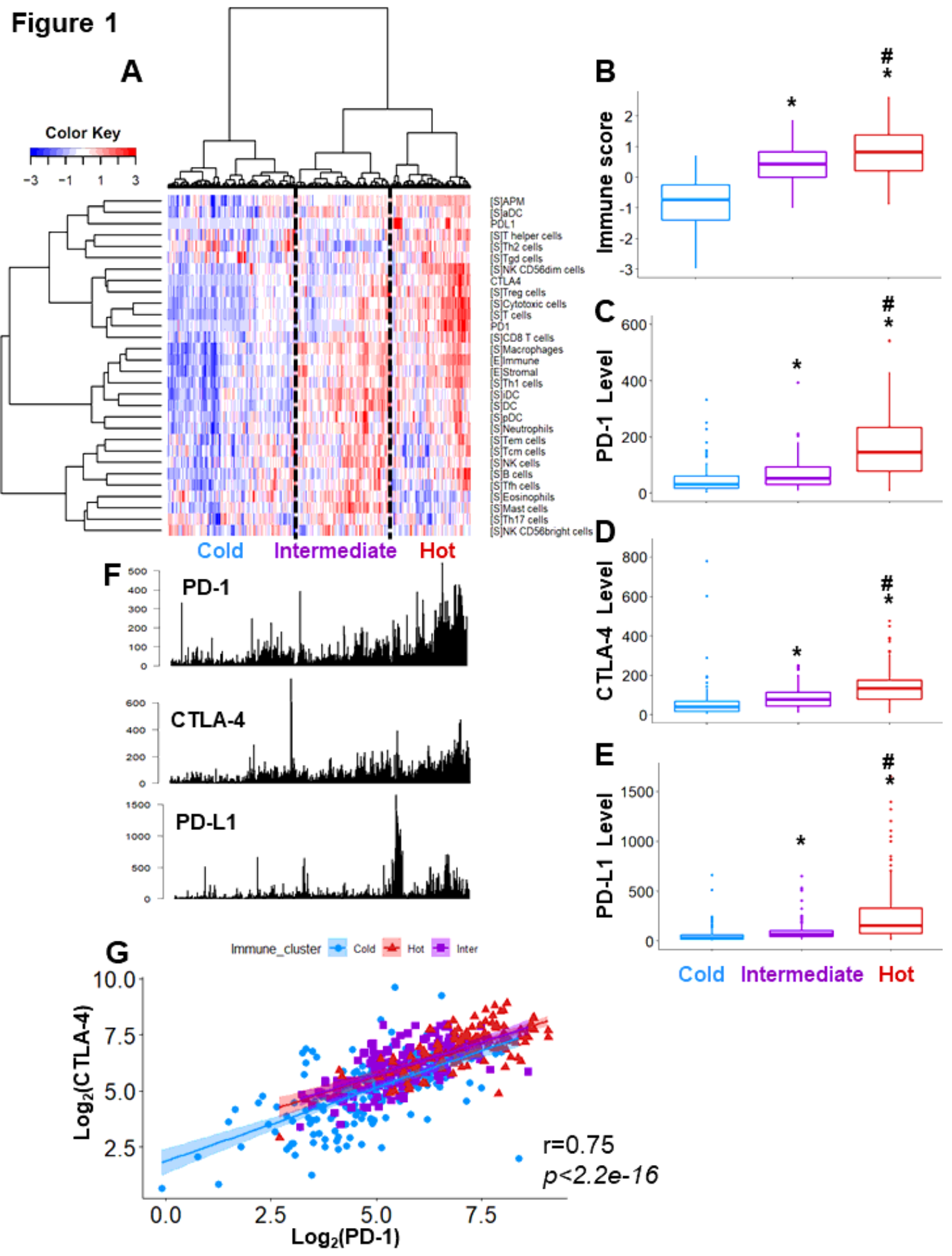

Figure 1

The tumor immune microenvironment (TIME) of lung adenocarcinoma (LUAD). (A) Unsupervised clustering analysis of 517 LUAD tumor samples from TCGA cohort depicting relative scores of 24 immune cell types, ESTIMATE-based immune and stromal scores, as well as relative expression values of PD-1, PD-L1, and CTLA-4. The tumors are clustered into three TIME subgroups (hot, intermediate, cold). [S]:ssGSEA-based score, [E]: ESTIMATE-based score. A key for the heatmap range is shown on the left. (B- 
E) Overall ESTIMATE immune scores (B), relative expression values of PD-1 (C), CTLA-4 (D), and PD-L1 (E) among hot, intermediate and cold tumors of LUAD. * $p<0.05$ when compared with the cold subgroup, $\# p<0.05$ when compared with the intermediate subgroup, unpaired $t$ tests. $(F)$ Bar plots representing the relative expression levels of PD-1 (top), CTLA-4 (middle), and PD-L1 (bottom). Each bar is plotted according to the clustered order from the heatmap result shown in $(A)$ and thus corresponds to each sample above. (G) A scatter plot depicting correlations between CTLA-4 and PD-1 expression in LUAD tumors. Each dot represents each sample from the LUAD cohort. Correlation analyses of each subtype of the cohort are also shown: hot (red), intermediate (purple), and cold (blue) tumors. Spearman correlation coefficient ( $r$ ) and $p$ values are computed between Log2 values of CTLA-4 and PD-1 expression. 


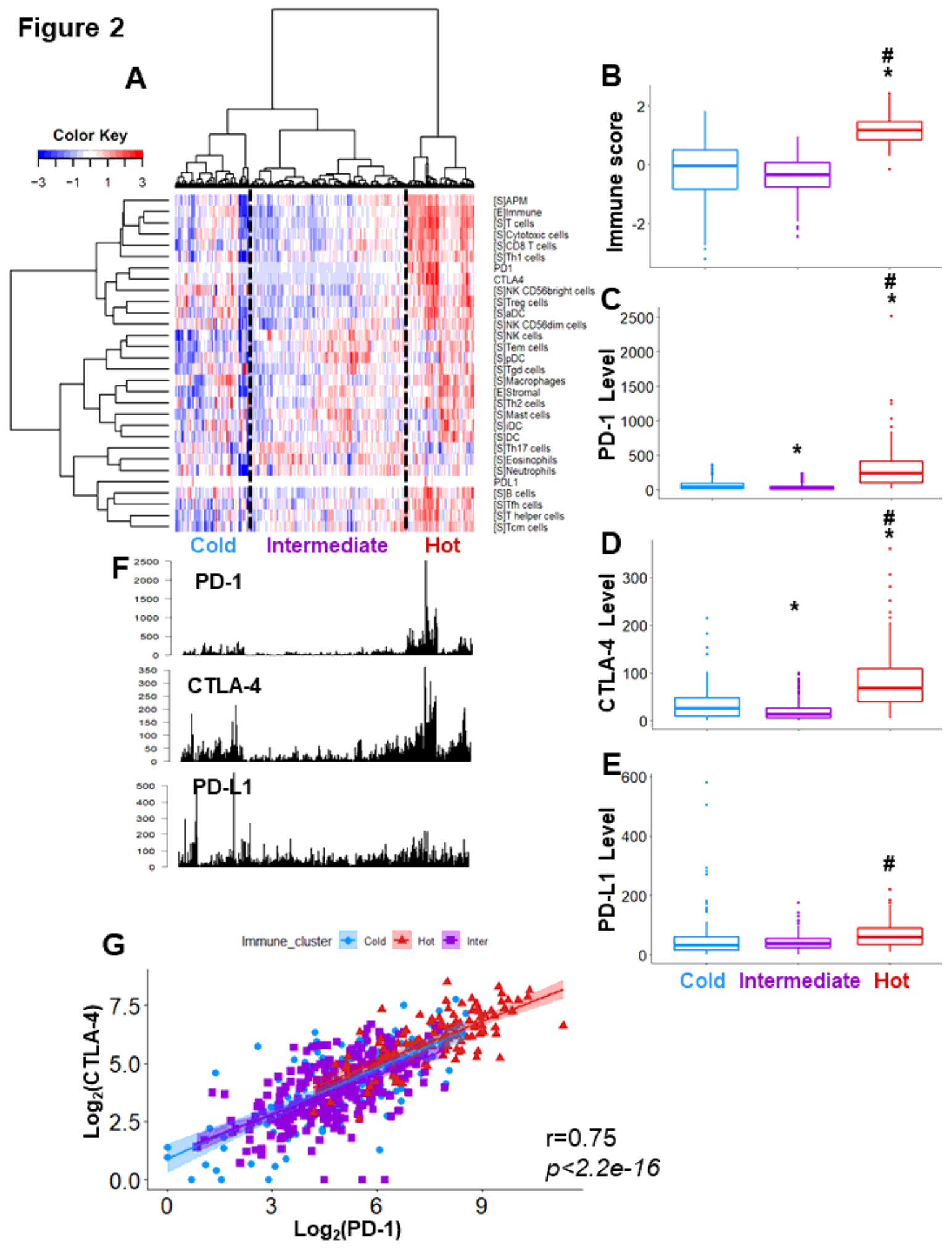

Figure 2

The TIME of kidney renal clear cell carcinoma (KIRC). (A) Unsupervised clustering analysis of $506 \mathrm{KIRC}$ tumor samples from TCGA cohort depicting relative scores of 24 immune cell types, ESTIMATE-based immune and stromal scores, as well as relative expression values of PD-1, PD-L1, and CTLA-4. The tumors are clustered into three TIME subgroups (hot, intermediate, cold). [S]:ssGSEA-based score, [E]: ESTIMATE-based score. A key for the heatmap range is shown on the left. (B-E) Overall ESTIMATE 
immune scores (B), relative expression values of PD-1 (C), CTLA-4 (D), and PD-L1 (E) among hot, intermediate and cold tumors of KIRC. ${ }^{*} p<0.05$ when compared with the cold subgroup, $\# p<0.05$ when compared with the intermediate subgroup, unpaired $t$ tests. $(\mathbf{F})$ Bar plots representing the relative expression levels of PD-1 (top), CTLA-4 (middle), and PD-L1 (bottom). Each bar is plotted according to the clustered order from the heatmap result shown in (A) and thus corresponds to each sample above. (G) A scatter plot depicting correlations between CTLA-4 and PD-1 expression in KIRC tumors. Each dot represents each sample from the KIRC cohort. Correlation analyses of each subtype of the cohort are also shown: hot (red), intermediate (purple), and cold (blue) tumors. Spearman correlation coefficient ( $r$ ) and $p$ values are computed between Log2 values of CTLA-4 and PD-1 expression. 


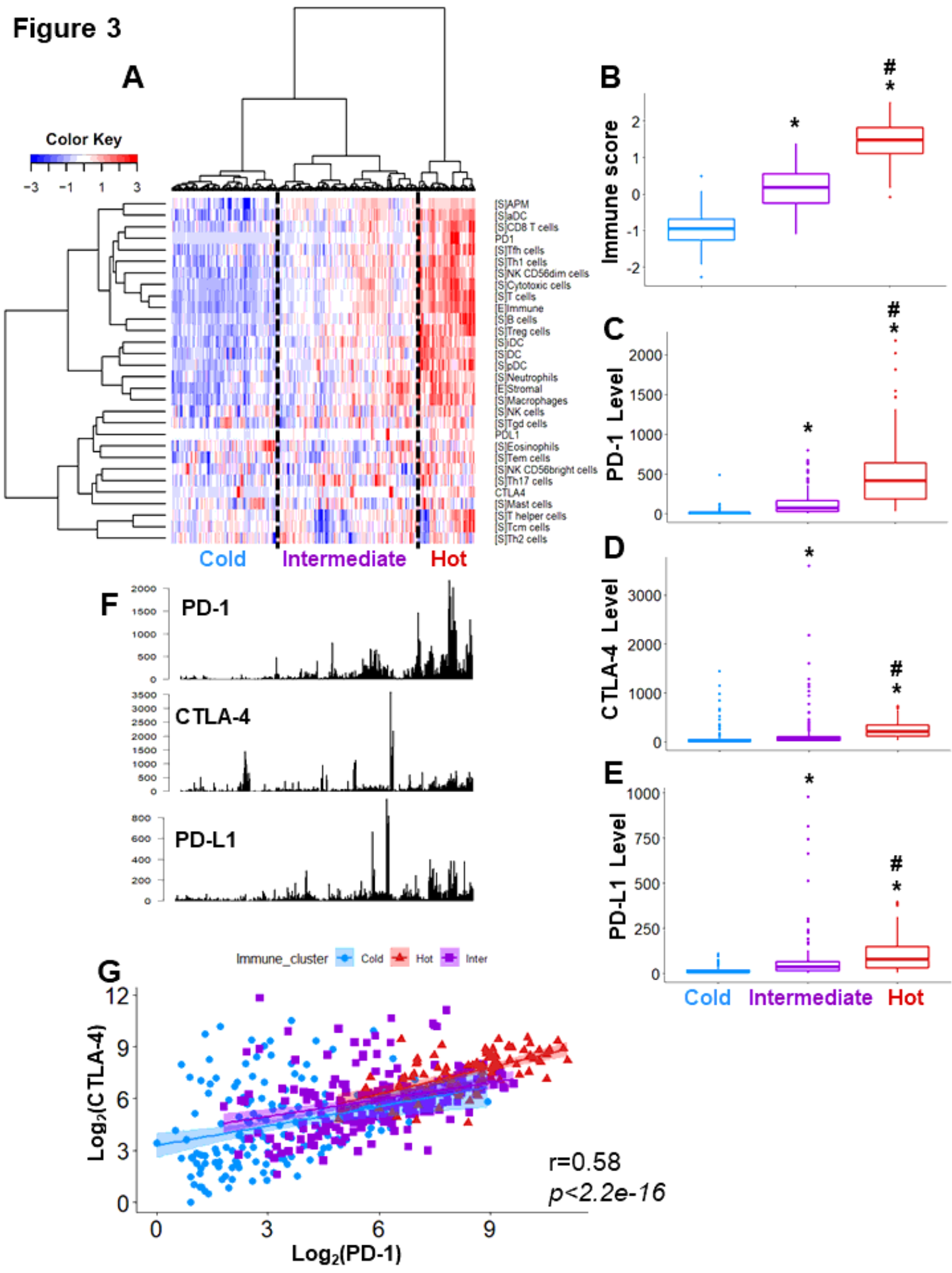

Figure 3

The TIME of skin cutaneous melanoma (SKCM). (A) Unsupervised clustering analysis of 472 SKCM tumor samples from TCGA cohort depicting relative scores of 24 immune cell types, ESTIMATE-based immune and stromal scores, as well as relative expression values of PD-1, PD-L1, and CTLA-4. The tumors are clustered into three TIME types (hot, intermediate, cold). [S]: ssGSEA-based score, [E]: ESTIMATE-based score. A key for the heatmap range is shown on the left. (B-E) Overall ESTIMATE 
immune scores (B), relative expression values of PD-1 (C), CTLA-4 (D), and PD-L1 (E) among hot, intermediate and cold tumors of SKCM. ${ }^{*} p<0.05$ when compared with the cold sub-group, $\# p<0.05$ when compared with the intermediate sub-group, unpaired $t$ tests. $(\mathbf{F})$ Bar plots representing the relative expression levels of PD-1 (top), CTLA-4 (middle), and PD-L1 (bottom). Each bar is plotted according to the clustered order from the heatmap result shown in (A) and thus corresponds to each sample above. (G) A scatter plot depicting correlations between CTLA-4 and PD-1 expression in SKCM tumors. Each dot represents each sample from the SKCM cohort. Correlation analyses of each subtype of the cohort are shown as well: hot (red), intermediate (purple), and cold (blue) tumors. Spearman correlation coefficient (r) and $p$ values are computed between Log2 values of CTLA-4 and PD-1 expression. 

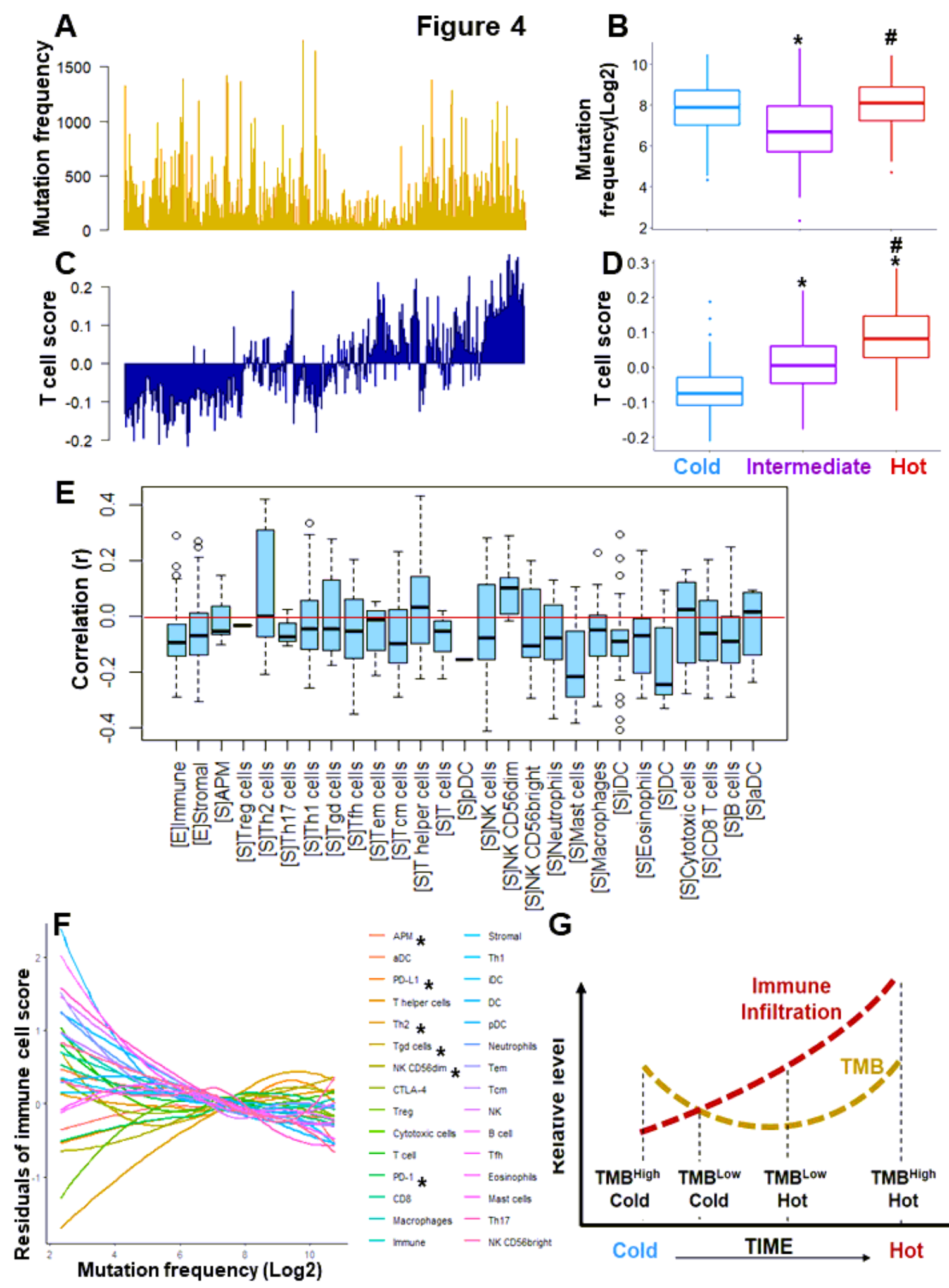

G

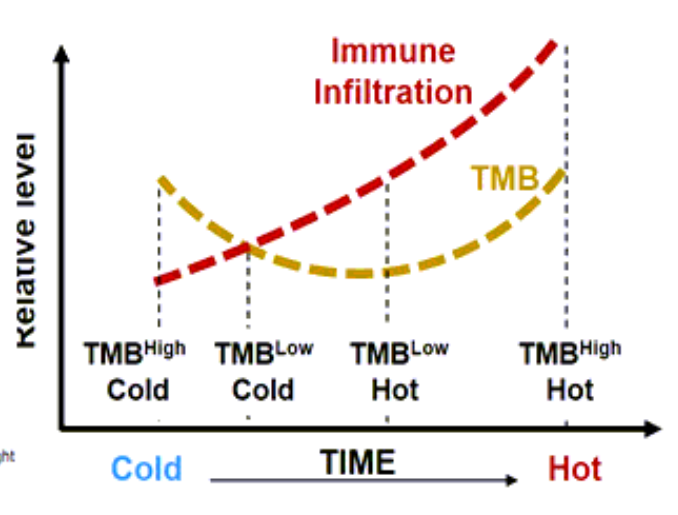

Figure 4

Tumor mutational burden (TMB) in LUAD does not correlate with immune cell infiltration in the tumor microenvironment. (A-B) Vertical bar plots (A) representing raw TMB values (the sum of somatic nonsynonymous mutations) of 517 LUAD tumors from TCGA cohort and their quantification in a Log2transformed form (B) among three TIME subtypes (hot, intermediate, cold). (C-D) Bar plots (C) showing the ssGSEA-based T cell scores of the LUAD tumors and their quantification (D) among the three TIME 
subtypes. Each bar shown in $A$ and $C$ is plotted according to the clustered order from the heatmap result shown in Fig $1 \mathrm{~A}$. * $p<0.05$ when compared with the cold subgroup, $\# p<0.05$ when compared with the intermediate subgroup, unpaired $t$ tests. $(E)$ One to one hundred forty one genes representing 24 immune cells, APM scores, or ESTIMATE immune\&stromal scores were selected, and the correlations with TMB (Log2-transformed) are shown. All $r$ values shown in the graphs are Spearman's coefficient values. (F) Correlation plots between TMB values (log2-based values of sum of the somatic nonsynonymous mutations) and the residuals of corresponding abundance of 30 immune features after linear regression against tumor purity. Statistical significance was evaluated using partial Spearman's correlation adjusted for tumor purity. *indicates an immune feature displaying a positive partial Spearman's correlation with statistical significance $(p<0.05)$ with TMB values. $(\mathbf{G})$ The proposed model depicting the relationship between $T$ cell-mediated immune responses and TMB in LUAD. The TIME of the cancer can be subdivided into four groups: immunologically hot, inflamed tumors with high (TMB ${ }^{\text {High }} \mathrm{Hot}$ ) or low (TMB $\left.{ }^{\text {Low }} \mathrm{Hot}\right)$ $\mathrm{TMB}$, and immunologically cold tumors with high (TMB ${ }^{\text {High }}$ Cold) or low (TMB ${ }^{\text {Low }}$ Cold) TMB. 
Figure 5
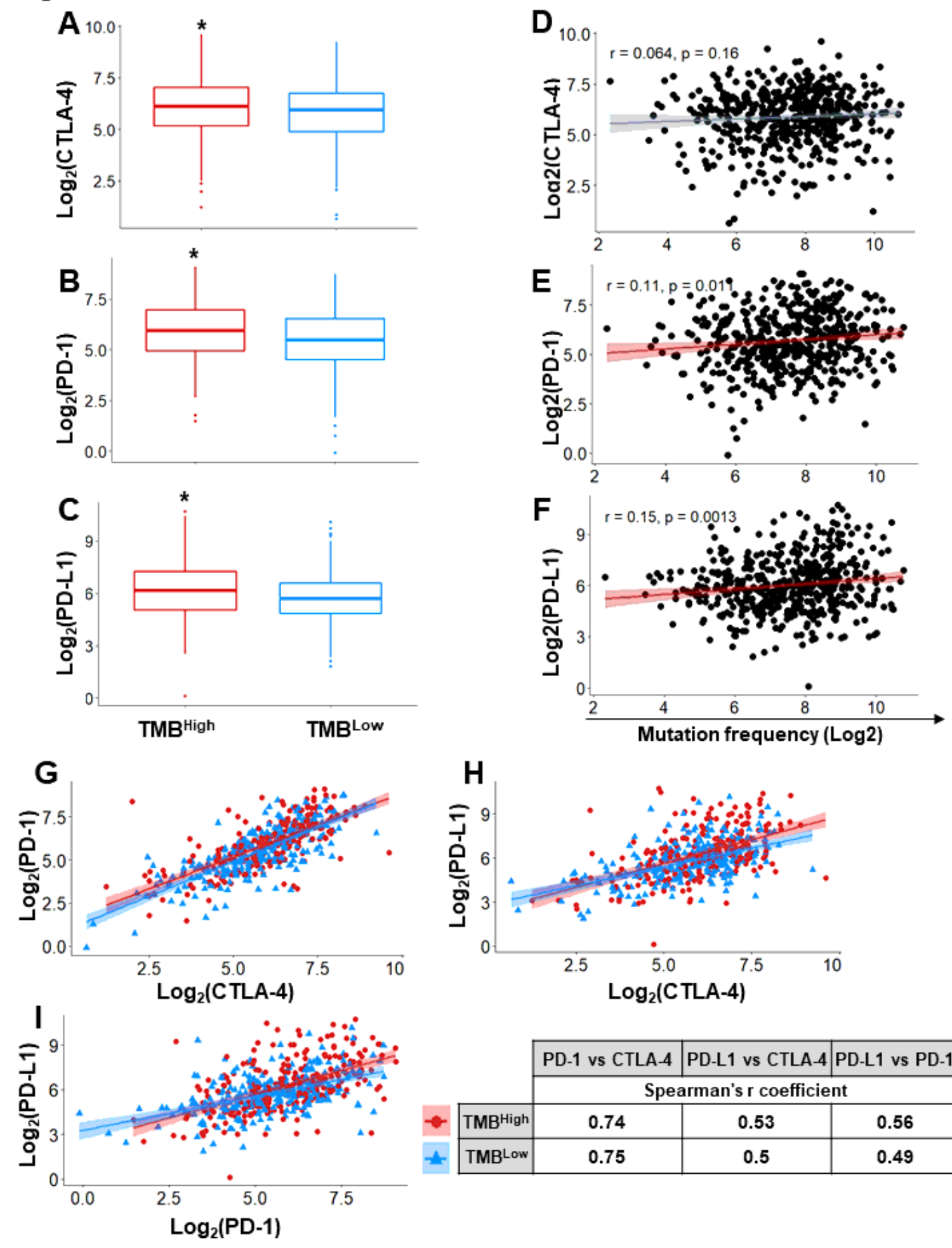

\begin{tabular}{|c|c|c|c|}
\cline { 2 - 4 } \multicolumn{1}{c|}{} & PD-1 vs CTLA-4 & PD-L1 vs CTLA-4 & PD-L1 vs PD-1 \\
\cline { 2 - 4 } \multicolumn{1}{c|}{} & \multicolumn{3}{|c|}{ Spearman's r coefficient } \\
\hline TMB $^{\text {High }}$ & 0.74 & 0.53 & 0.56 \\
\hline TMB $^{\text {Low }}$ & 0.75 & 0.5 & 0.49 \\
\hline
\end{tabular}

\section{Figure 5}

The associations between immune checkpoints do not depend on TMB levels in LUAD. (A-C) Levels of CTLA-4 (A), PD-1 (B), or PD-L1 (C) expression in TMB ${ }^{\text {High }}$ (>median) and TMB ${ }^{\text {Low }}$ ( $\leq$ median) tumor samples in the LUAD cohort. ${ }^{*} p<0.05$, unpaired $t$ test. (D-F) Scatter plots representing correlations between TMB (Log2-transformed) and CTLA-4 (D), PD-1 (E), or PD-L1 (F) expression in LUAD tumor samples. Expression values of CTLA-4, PD-1 and PD-L1 are log base 2-transformed. All $r$ values in the 
graphs represent Spearman's coefficient values. (G) Scatter plots showing correlation between CTLA-4 and PD-1 levels (Log2 values) in TMB ${ }^{\text {High }}$ (>median, red dots) and TMB ${ }^{\text {Low }}$ ( $\leq$ median, blue dots) LUAD tumors. $(\mathrm{H})$ Scatter plots showing correlation between CTLA-4 and PD-L1 levels (Log2 values) in TMB High (>median, red dots) and TMB ${ }^{\text {Low }}$ ( $\leq$ median, blue dots) LUAD tumors. (I) Scatter plots showing correlation between PD-L1 and PD-1 levels (Log2 values) in TMB ${ }^{\text {High }}$ (>median, red dots) and TMB ${ }^{\text {Low }}$ ( $\leq$ median, blue dots) LUAD tumors. All $r$ values shown in the graphs represent Spearman's coefficient values. $P$ values for all correlations are $p \leq 1.1 e-15$.

\section{Supplementary Files}

This is a list of supplementary files associated with this preprint. Click to download.

- SupplementaryFigure1EPMA.tif

- SupplementaryFigure2EPMA.tif

- SupplementaryFigure3EPMA.tif

- SupplementaryTable1EPMA.tif

- SupplementaryTable2EPMA.tif 